\title{
Comparison of Nearest Neighbor (ibk), Regression by Discretization and Isotonic Regression Classification Algorithms for Precipitation Classes Prediction
}

\author{
Solomon Mwanjele \\ Mwagha \\ Taita Taveta University College \\ P.O.Box 308-80300 \\ Voi Kenya
}

\author{
Masinde Muthoni \\ Central University of \\ Technology \\ Bloemfontein \\ South Africa
}

\author{
Peter Ochieg \\ Taita Taveta University College \\ P.O.Box 635-80300
}

\begin{abstract}
Selection of classifier for use in prediction is a challenge. To select the best classifier comparisons can be made on various aspects of the classifiers. The key objective of this paper was to compare performance of nearest neighbor (ibk), regression by discretization and isotonic regression classifiers for predicting predefined precipitation classes over Voi, Kenya. We sought to train, test and evaluate the performance of nearest neighbor (ibk), regression by discretization and isotonic regression classification algorithms in predicting precipitation classes. A period of 1979 to 2008 daily Kenya Meteorological Department historical dataset on minimum/maximum temperatures and precipitations for Voi station was obtained. Knowledge discovery and data mining method was applied. A preprocessing module was designed to produce training and testing sets for use with classifiers. Isotonic Regression, K-nearest neighbours classifier, and RegressionByDiscretization classifiers were used for training training and testing of the data sets. The error of the predicted values, root relative squared error and the time taken to train/build each classifier model were computed. Each classifier predicted output classes 12 months in advance. Classifiers performances were compared in terms of error of the predicted values, root relative squared error and the time taken to train/build each classifier model. The predicted output classes were also compared to actual year classes. Classifier performances to actual precipitation classes were compared. The study revealed that the nearest neighbor classifier is a suitable for training rainfall data for precipitation classes prediction.
\end{abstract}

\section{General Terms}

Clasification Algorithms, Data Mining, Knowledge Discovery

\section{Keywords}

Regression by discretization, isotonic regression, nearest neighbor(ibk), precipitation prediction, classification algorithms, classifier performance

\section{INTRODUCTION}

There has been a lot of research aimed at precipitation predictions over selected regions where solutions were based on traditional, statistical and modern computational methods or a combination. Though these precipitation predictions were useful in overall region rainfall picture depiction, challenges exist for the prediction of quantity classes of precipitation for fixed range durations for instance weekly or monthly quantities in a year. Successful prediction of precipitation in fixed range durations can aid in selection of activities during rainy seasons such as cropping where different crops require different water requirements, or selection of a grazing land for nomadic pastoralists for a particular duration of time.

Classification algorithms continue to play a big role in prediction of events based on historical data. In order to predict precipitation classes in advance algorithm performances must compared and the best one selected.

\section{LITERATURE REVIEW}

A study on drought forecasting [2] analyzed rainfall frequencies using data from 248 rain gauges (1938-2005). SPI was determined using ANN feed feedfoward and back propagation algorithm. The findings showed that the result of ANN is suitable for drought forecast. Another study aimed at comparing ANN and ANFIS in precipitation prediction [2] realized ANN efficient in rain prediction. predicting agricultural drought [12] was done using 1880-2005 rain data to analyze agricultural drought. By applying fuzzy sets analysis on the condition of crops and valid rain history, result of fuzzy clustering obtained. Drought years extracted from fuzzy clustering results. Time series used to predict next drought year. A study in [8] was aimed at translating seasonal forecast to agricultural terms using crop simulation model to translate seasonal forecast to agricultural terms. The results offered support to farmer's climate risk management. In China rainfall was predicted by direct determination of surface soil moisture using microwave observation [11] where data was acquired and analyzed over several test sites. The study was validated by conducted large field experiments. Agricultural drought was predicted in paddy fields using remotely sensed data [7] where NDVI was found to be reasonable in detecting agricultural drought. The study was limited by insufficient data as fuzzy was done in non cropping time. Metrological conditions causing drought were evaluated using the differentiate between precipitation \& evapotranspiration to evaluate metrological conditions causing drought [4]. In USA historical patterns for drought were identified using VegOut Model that integrated Climate Ocean, satellite indicators[10]; regression trees were used to identify historical patterns for drought intensely and vegetation. SPI and PDSI were used to represent climate vulnerability. This study was evaluated using 2006 drought year. Unlike previous studies this paper contributes on prior work by considering crop production history and weather data history together with classification algorithms to come up with precipitation classes. Our work provides future classes projections with a limit of twelve months in advance predictions. By borrowing from previous studies this research 
emphasis is on comparison of classification algorithms in rainfall prediction in order to select the best.

\section{METHODOLOGY}

A period of 1979 to 2008 daily KMD historical dataset on minimum/maximum temperatures and precipitations for Voi KMD station was obtained. Next the knowledge Discovery and Data mining (KDD) process steps were applied. A preprocessing module was designed to produce training and testing sets of files for use with the classifiers. Three classifiers (isotonic regression, k-nearest neighbours classifier, and regression by discretization) were used for training training and testing of the data sets. A knowledge flow was implemented for each of the three classifiers. The Waikato Environment for Knowledge Analysis (WEKA) and Java programming environment (JCreator and Net Beans) were used.

\section{RESULTS}

A preprocessing module was designed to produce two sets of files for use with the Weka Knowledge flow each with five attributes namely:-

Year,

Month,

Scaled precipitation values (range: 0 to 1 ),

Precipitation class values,

Index class (range: -2 to 2 ).

Three classifiers (Isotonic Regression, K-nearest neighbours classifier, and RegressionByDiscretization) were considered for training training and testing of the data sets. The classifiers produced output classes with the following attributes:-

Precipitation class values with removal filtered applied,

Index class identified as the class variable.

The output of each of the classifiers is as follows:-

Isotonic Regression

This algorithm learns an isotonic regression model to pick the attribute that result in the lowest squared error. It does not allow missing values and can only deal with numeric attributes. It considers the monotonically increasing case as well as the monotonically decreasing case.

The running information on using this classifier is as follows:-

Scheme: weka.classifiers.functions.IsotonicRegression

Relation: training-

weka.filters.unsupervised.attribute.Remove-R4

Instances: 372

Attributes: 4

year
month
scaled_prec
index_class

Test mode: 10 -fold cross-validation

$===$ Classifier model (full training set) ===
Isotonic regression

Based on attribute: scaled_prec

$\begin{array}{llll}\text { prediction: } & -2 & \text { cut point: } & 0 \\ \text { prediction: } & -1.5 & \text { cut point: } & 0.08 \\ \text { prediction: } & -1 & \text { cut point: } & 0.11 \\ \text { prediction: } & -0.5 & \text { cut point: } & 0.19 \\ \text { prediction: } & 0 & \text { cut point: } & 0.37 \\ \text { prediction: } & 0.5 & \text { cut point: } & 0.46 \\ \text { prediction: } & 1 & \text { cut point: } & 0.56 \\ \text { prediction: } & 1.5 & \text { cut point: } & 0.65 \\ \text { prediction: } & 2 & & \end{array}$

prediction: 2

Time taken to build model: 0.03 seconds

$===$ Cross-validation $===$
$===$ Summary $===$

Correlation coefficient

Class complexity | order 0

bits 2.5057 bits/instance

Class complexity | scheme 24.4368 bits 0.0657 bits/instance

Complexity improvement (Sf) 907.6867 2.44 bits/instance

Mean absolute error

Root mean squared error

Relative absolute error

Root relative squared error

Total Number of Instances

The error of the predicted values for numeric classes is 0.0027

The root relative squared error is $3.7127 \%$

K-nearest neighbor classifier

$\mathrm{K}$-nearest neighbour classifier can select appropriate value of $\mathrm{K}$ based on cross-validation. It can also do distance weighting using a simple distance measure to find the training instance closest to the given test instance, and predicts the same class as this training instance. If multiple instances are the same (smallest) distance to the test instance, the first one found is used.

The running information for this classifier is as follows:-

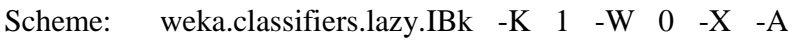
"weka.core.neighboursearch.LinearNNSearch -A ।"weka.core.EuclideanDistance -R 3,4।" -P"

Relation: trainingweka.filters.unsupervised.attribute.Remove-R4

Instances: 372

Attributes: 4

year

month 


$$
\text { scaled_prec }
$$

index_class

Test mode: 10-fold cross-validation

$===$ Classifier model (full training set) $===$

IB1 instance-based classifier

Using 1 nearest neighbour(s) for classification

Time taken to build model: 0 seconds

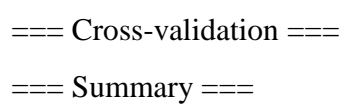

Correlation coefficient

0.9993

Class complexity | order 0

2.5178 bits/instance

Class complexity | scheme

0.0618 bits/instance

Complexity improvement (Sf)

936.637

bits

2.456 bits/instance

Mean absolute error

bits

Root mean squared error

Relative absolute error

Root relative squared error

Total Number of Instances

The error of the predicted values for numeric classes is 0.0.0027

The root relative squared error is $3.7048 \%$

\section{Regression By Discretization}

Regression by discretization is a scheme that employs any classifier on a copy of the data that has the class attribute (equal-width) discretized. The predicted value is the expected value of the mean class value for each discretized interval (based on the predicted probabilities for each interval).

The base classifier used is J48 Class for generating a pruned or unpruned $\mathrm{C} 4.5$ decision trees.

The output of this classifier is as follows:-

Scheme: weka.classifiers.meta.RegressionByDiscretization -B 10 -W weka.classifiers.trees.J48 -- -C 0.25 -M 2

Confidence factor for pruning is 0.25 , to use binary splits and restrict the minimum number of instances in a leaf to 2 (grow the tree fully).

Relation:

training-

weka.filters.unsupervised.attribute.Remove-R4

The name of the relation contains in it the name of data file used to build it, and the names of filters that removes the fourth attribute

Instances: 372

Attributes: 4

$$
\text { year }
$$$$
\text { month }
$$

$$
\begin{aligned}
& \text { scaled_prec } \\
& \text { index_class }
\end{aligned}
$$

Test mode: 10-fold cross-validation

$===$ Classifier model (full training set) $===$

Regression by discretization

Class attribute discretized into 10 values

Classifier spec: weka.classifiers.trees.J48 -C 0.25 -M 2

J48 pruned tree

$$
\text { scaled_prec }<=0.073766
$$

| scaled_prec <=0: '(-inf--1.6]' (45.0)

| scaled_prec >0: '(-1.6--1.2]' (177.0)

scaled_prec $>0.073766$

| scaled_prec $<=0.186199$

| | scaled_prec <=0.107971: '(-1.2--0.8]' (19.0)

| | scaled_prec >0.107971: '(-0.8--0.4]' (40.0)

| scaled_prec > 0.186199

| | scaled_prec <=0.364366: '(-0.4-0]' (47.0)

| | scaled_prec > 0.364366

| | | scaled_prec $<=0.555324$

| | | | scaled_prec <=0.447353: '(0.4-0.8]' (12.0)

｜｜｜｜ scaled_prec > 0.447353: '(0.8-1.2]' (12.0)

| | | scaled_prec $>0.555324$

| | | | scaled_prec <=0.650506: '(1.2-1.6]' (14.0)

｜｜｜ scaled_prec >0.650506: '(1.6-inf)' (6.0)

Number of Leaves: 9

Size of the tree: 17

Time taken to build model: 0.05 seconds

$===$ Cross-validation $===$
$===$ Summary $===$

Correlation coefficient

0.9974

Class complexity | order 0 936.637 bits

2.5178 bits/instance

Class complexity | scheme

71.8388

bits 0.1931 bits/instance

Complexity improvement (Sf) $864.7982 \quad$ bits 2.3247 bits/instance

Mean absolute error

0.0108

Root mean squared error

Relative absolute error

$1.3101 \%$

Root relative squared error

$7.4096 \%$

Total Number of Instances

The error of the predicted values for numeric classes is 0.0108 The root relative squared error is $7.4096 \%$ 
Evaluation

Comparison of classifiers 2009 predictions to 2009 actual precipitation classes

\begin{tabular}{|c|c|c|c|c|}
\hline \multirow[t]{3}{*}{ Month } & \multirow[t]{3}{*}{ Actual 2009} & \multicolumn{3}{|c|}{2009 Class predicted by classifies } \\
\hline & & \multicolumn{2}{|l|}{ Ibk } & \multirow{2}{*}{$\begin{array}{l}\text { Isotonic } \\
5 \text { Step }\end{array}$} \\
\hline & & 5 Step & 10 Step & \\
\hline Jan & -0.5 & 2 & -1 & 2 \\
\hline Feb & -1.5 & -1 & -1.5 & -0.5 \\
\hline Mar & -1.5 & -0.5 & 0 & -0.5 \\
\hline Apr & 0.5 & 0 & 2 & 0.5 \\
\hline May & -2 & -1.5 & -1.5 & -2 \\
\hline Jun & -1.5 & -1.5 & -1.5 & -1.5 \\
\hline Jul & -1.5 & -2 & -2 & -1.5 \\
\hline Aug & -1.5 & -1.5 & -1.5 & -2 \\
\hline Sep & -2 & -1.5 & -1.5 & -2 \\
\hline Oct & 0 & 0 & -1.5 & -0.5 \\
\hline Nov & 0 & 0 & 1.5 & 0 \\
\hline Dec & 1.5 & 0.5 & 1.5 & 0.5 \\
\hline \multicolumn{2}{|c|}{ Error of the predicted values } & \multicolumn{2}{|l|}{0.0027} & 0.0027 \\
\hline \multicolumn{2}{|c|}{ Root relative squared error } & \multicolumn{2}{|c|}{$3.7048 \%$} & $3.7127 \% \mathrm{n}$ \\
\hline \multicolumn{2}{|c|}{ Time taken to train/build model } & \multicolumn{2}{|c|}{0 seconds } & 0.03 secons \\
\hline
\end{tabular}

Comparison of output classes from classifiers

Graph of classifiers 2009 predictions outputs compared to 2009 actual

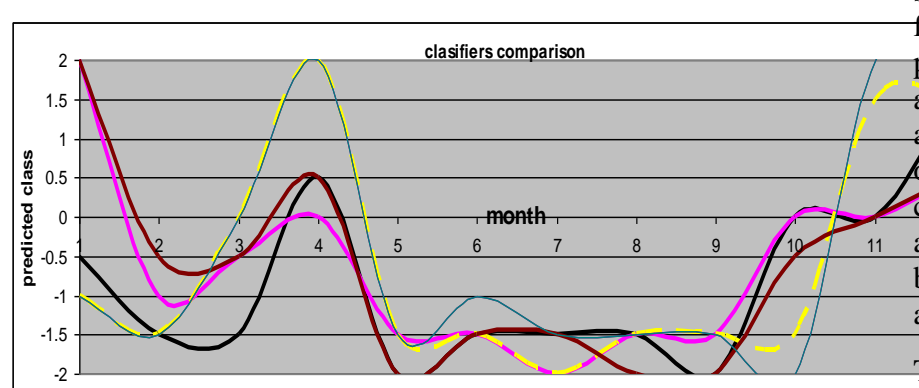

\section{CONCLUSION}

The precipitation class prediction output results obtained showed that the nearest neighbor classifier is a suitable tool for training meteorological data for precipitation classes. As part of machine learning the IBk classifier results acgomplished intelligence through the knowledge discovery and data mining process as aimed in the study major objective. Evaluation of our study results shows that the Ibk classiffer had the least error of the predicted values $(0.0027)$ ande the least root relative squared error $(3.7048 \%)$ hence can be used to predict precipitation in advance with greater accuracy compared to the other two classifiers.

The recommendation for designing a solution that can cater for precipitation predictions in multiple regions is open for this study as future work. In Kenya for instance all districts can be represented and in precipitation classes predicted. Finally with adjustments of our prediction predictions socioeconomic measures on droughts anticipations can be suggested e.g. early warning allowing systems can be developed.

Figure 49: graph comparing classifiers outputs on 5 year and 10 year sample step with actual

In figure 55 below the predicted classes are compared to the actual computed for 2009. The predictions correspond to most of the monthly actual classes. The predictions were based on IBk classifier with 10 year step sampling.

\section{REFERENCES}

[1] Ashok, M. et al, 2006. Linking Seasonal Climate Forecasts with Crop Simulation to Optimize Maize Management, CCSP Workshop: Climate Science in Support of Decision Making, 14-16 November 2005 Crystal Gateway Marriott Arlington, Virginia 14-16 November 2005

[2] Dostrani, M. et al (2010). Application of ANN and ANFIS Models on Dryland Precipitation Prediction (Case Study: Yazd in Central Iran). Journal of Applied Sciences, 10: 2387-2394.

[3] Gong, Z. et al, 2010. Risk Prediction of Agricultural Drought in China. 2010 Seventh International Conference on Fuzzy Systems and Knowledge Discovery (FSKD 2010).Kenya Meteorological Department, Agrometeorological bulletin, Issue No. 27/2009. 
[4] Kozyra, J. et al 2009. Institute Of Soil Science and Plant Cultivation National Research Institute, International Symposium, Climate change and Adaptation Options in Agriculture, Viena, June, 22-23 2009.

[5] Ladislaus B. et al, 2010. Indigenous knowledge in seasonal rainfall prediction in Tanzania: A case of theSouth-western Highland of Tanzania, Journal of Geography and Regional Planning Vol. 3(4), pp. 6672,April 2010.

[6] Lin Zhu, Jing M. Chen, Qiming Qin, Mei Huang, Lianxi Wang, Jianping Li, Bao Cao: Assimilating Remote Sensing based Soil Moisture in an Ecosystem Model (BEPS) for Agricultural Drought Assessment.IGARSS (5) 2008: 437-440

[7] Niu Shulian; Susaki Junichi, 2006. Detection of Agricultural Drought in Paddy Fields Using NDVI from MODIS Data. A Case Study in Burirum Province, Thailand.
[8] Patrick O, 2006. Agricultural Policy in Kenya: Issues and Processes, A paper for the Future Agricultures Consortium workshop, Institute of Development Studies, 20-22 March 2006.

[9] Peter Reutemann, (2007). WEKA Knowledge Flow Tutorial for Version 3-5-7, University of Waikato 2007

[10] Tsegaye T. \& Brian W. 2007. The Vegetation Outlook (VegOut): A New Tool for Providing Outlooks of General Vegetation Conditions Using Data Mining Techniques. ICDM Workshops 2007: 667-672

[11] Z. (Bob) Su, Y. Chen, M. Menenti, J. Sobrino, Z.-L. Li, W. Verhoef, L. Wang, Y. Ma, L. Wan, Y. He, Q.H.

[12] Liu, C. Li, J. WEN, R. van der Velde, M. van Helvoirt, W. Lin, X. Shan, 2007. Drought Monitoring and Prediction over China, In: Proceedings of the 2008 Dragon symposium, Dragon programme, final results, 2004-2007, Beijing, China 21-25 April 2008. 